Future of German

\section{neutron source}

\section{hangs in the balance}

[MUNICH] The planned opening in 2001 of a controversial 20-MW research reactor being built by the Technical University of Munich at Garching could depend on the outcome of discussions being held this week by Germany's new coalition government on its nuclear policy.

In particular, the discussions will address a proposal from Green environment minister Jürgen Trittin to bar the licensing of new nuclear reactors - including research reactors used as a source of neutrons for medical and material sciences - of more than $1 \mathrm{MW}$ thermal power.

The reactor concerned, FRM II, has been strongly opposed by environmental groups because it has been designed to burn bombgrade, highly enriched uranium (HEU), in defiance of a 1978 international anti-nuclear proliferation agreement that all reactors should convert, if technically possible, to low enriched uranium.

An amendment to this agreement requires the United States to take back HEU waste from reactors agreeing to convert (see Nature $370,90 ; 1994)$. The Technical University overcame opposition to win building licences, and the DM770 million (US\$456 million) building operation is well under way. But the reactor requires an operation licence, for which the university must apply next year.

Trittin's proposal, leaked to the press last week, also includes plans to bar the transport or reprocessing of nuclear waste in Germany. He argues that waste should be stored above ground at interim storage sites close to the reactors themselves. But this proposal has concerned research reactor operators.

The Hahn Meitner Institute in Berlin, for example, whose reactor is converting to LEU, was recently forced to send a consignment of spent fuel rods to the UK reprocessing facility in Dounreay, Scotland, because the US agreement to take back spent fuel was in temporary abeyance and its own limited storage facilities were dangerously overloaded (see Nature 377, 470; 1995).

If the shipped fuel has to be returned, it could cause a major dilemma. A spokesman for the institute says plans to build a new storage facility would take years to gain approval. Moreover, he says, the implications are unclear for spent fuel recently shipped to the United States, as US officials have not decided whether it should be stored or reprocessed.

A spokeswoman for the German research ministry says this week's talks are intended to clarify such issues. Meanwhile, the Technical University has mounted a vigorous press campaign opposing any decision to prevent its reactor from opening.

Alison Abbott

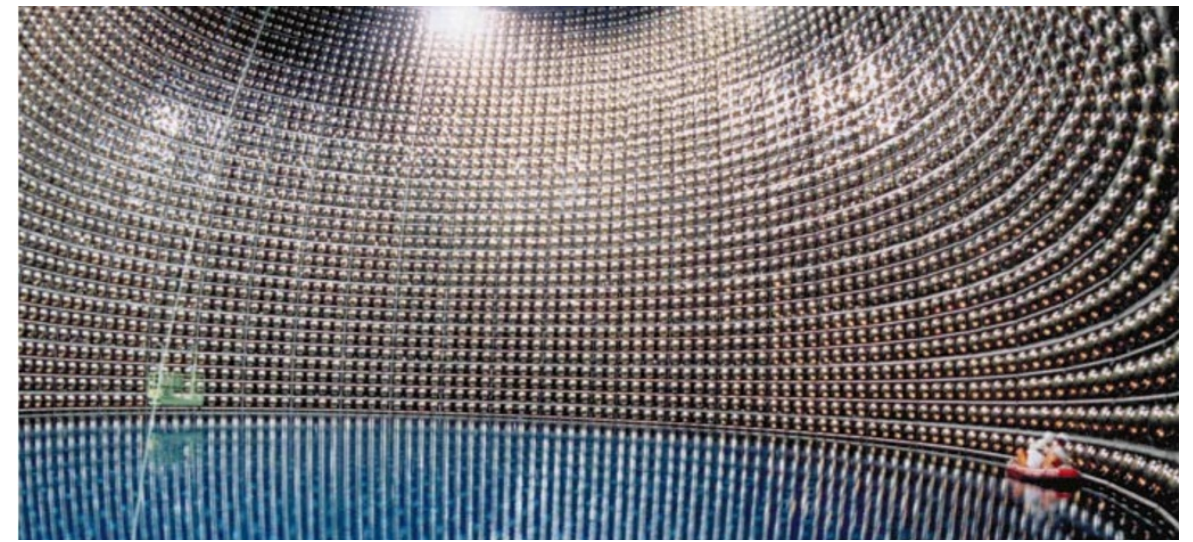

The Super-Kamiokande neutrino detector: results will throw light on cosmological theories.

\title{
Japanese centre will study the origins of the Universe
}

[TOKYO] The University of Tokyo has announced plans to set up a research centre to study the 'Big Bang' model of the origins of the Universe from both observational and theoretical perspectives.

One goal of the centre could be to explore a new and more flexible theory for solving the problems posed by the presence of free parameters in this model which has long restricted its predictive power.

Researchers from the university say their interest in the theory has been strengthened by recent findings from distant supernova explosions which suggest that the Universe entered a period of intense inflation shortly after the Big Bang, as advocated by the theory of 'cosmological inflation'.

The centre will be set up in April within the university's department of science. It will replace the Research Centre for the Early Universe (RESCEU), a five-year project funded by the Ministry of Education, Science, Sports and Culture (Monbusho) to study the origin and evolution of the Universe.

Although RESCEU, which began in 1995, should not end until March next year, its replacement by the new research centre will be a year earlier than scheduled. This is to allow the earliest possible start to the new project, which will merge five research divisions from RESCEU with three new divisions, including one set up specifically for overseas researchers.

The centre, which will conduct research in areas such as the very early Universe, dark matter, cosmic rays and the formation and evolution of galaxies, will be part of Monbusho's 'centre of excellence' programme, which supports research organizations carrying out 'highly creative scientific research'.

"An early start will allow us to take a step towards our aim to create an international base for research on the Big Bang model," says Katsuhiko Sato, professor of theoreti- cal astrophysics and the director of the new centre.

"We feel this is the time to give substance to the theoretical model by applying results from areas such as nuclear and particle physics as well as data obtained from the latest observations."

According to Sato, the centre will consist of two sections: theories of the early Universe, which will take a 'top down' approach based on the theory of cosmological inflation developed by Guth, Linde and Steinhardt in the early 1980s, which proposes a very rapid expansion of the Universe shortly after the Big Bang; and a 'bottom up' approach of creating models using observational data and results obtained from experiments in particle physics.

It is hoped that the observations of neutrinos from supernova explosions, such as those by researchers from Tokyo University's Institute for Cosmic Ray Research using the Super-Kamiokande neutrino detector, will contribute to the systematic study of particles needed to understand the early Universe.

Studies of the early Universe, including research on its birth and on cosmological phase transitions, primordial nucleosynthesis, and baryogenesis (the production of protons and neutrons), based on general relativity and particle physics, will use observational data obtained from national sources, such as Monbusho's Institute of Space and Astronomical Sciences, as well as those from international sources such as the Sloan Digital Sky Survey.

"The importance of the project lies in the fact that our research will be driven by both theory and observational data. This will allow quantitative comparisons between results from observations and those from theories, and will also help to integrate research results from different fields," says Sato.

AsakoSaegusa 